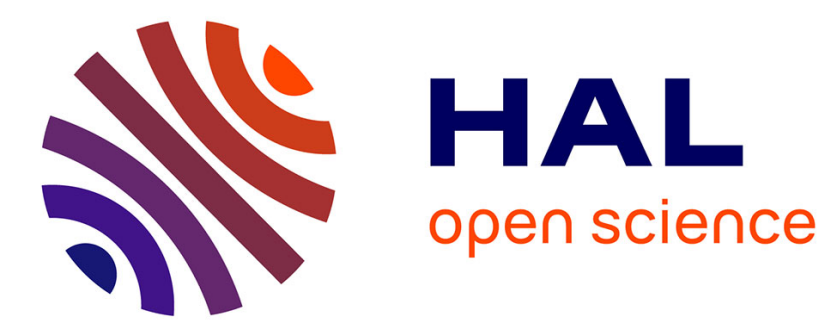

\title{
Surface plasmons in layered structures with semiconductor and metallic films
}

\author{
A.G. Schuchinsky, X. Yan
}

\section{To cite this version:}

A.G. Schuchinsky, X. Yan. Surface plasmons in layered structures with semiconductor and metallic films. European Physical Journal: Applied Physics, 2009, 46 (3), pp.1-13. 10.1051/epjap/2009021. hal-00480151

\section{HAL Id: hal-00480151 \\ https://hal.science/hal-00480151}

Submitted on 3 May 2010

HAL is a multi-disciplinary open access archive for the deposit and dissemination of scientific research documents, whether they are published or not. The documents may come from teaching and research institutions in France or abroad, or from public or private research centers.
L'archive ouverte pluridisciplinaire HAL, est destinée au dépôt et à la diffusion de documents scientifiques de niveau recherche, publiés ou non, émanant des établissements d'enseignement et de recherche français ou étrangers, des laboratoires publics ou privés. 


\title{
Surface plasmons in layered structures with semiconductor and metallic films
}

\author{
A. G. Schuchinsky, X. Yan \\ Queen's University Belfast, Belfast, BT3 9DT, UK, e-mail: a.schuchinsky@qub.ac.uk
}

\begin{abstract}
The complete spectrum of eigenwaves including surface plasmon polaritons (SPP), dynamic (bulk) and complex waves in the layered structures containing semiconductor and metallic films has been explored. The effects of loss, geometry and the parameters of dielectric layers on the eigenmode spectrum and, particularly, on the SPP modes have been analysed using both the asymptotic and rigorous numerical solutions of the full-wave dispersion equation. The field and Poynting vector distributions have been examined to identify the modes and elucidate their properties. It has been shown that losses and dispersion of permittivity qualitatively alter the spectral content and the eigenwave properties. The SPP counter-directional power fluxes in the film and surrounding dielectrics have been attributed to vortices of power flow, which are responsible for the distinctive features of SPP modes. It has been demonstrated for the first time that the maximal attainable slow-wave factor of the SPP modes guided by thin Au films at optical frequencies is capped not by losses but the frequency dispersion of the actual Au permittivity.
\end{abstract}

\section{Introduction}

Surface plasmon polaritons (SPP) at interfaces of lossy metallic and semiconductor layers and heterostructures have been extensively studied in the past - see, e.g., [1]-[10]. Recently this topic has regained considerable attention owing to the novel potential applications of the SPPs in near field imaging, and photonic and opto-electronic devices, see [11][13] and references therein. Although the basic theory of SPP had been developed long ago, majority of the results were obtained with the aid of the quasi-static approximations based upon the asymptotic solutions of the dispersion equations in the vicinities of plasmonic resonances. The effect of loss was taken into account either as small perturbations or through complex permittivity of the films in the approximate dispersion relationships [1], [2], [4]-[8]. Such an approach has provided an adequate qualitative description of the SPP properties in low loss media near the plasmonic resonances where the long-range and short-range SPP [5]-[7] in metallic films had been usually analysed at isolated frequencies. However, these approximations proved to be insufficient for the analysis of thin films with strong near field interactions of the interfaces and the finite structures where the detailed knowledge of the complete spectrum of eigenwaves becomes critical. Also, the actual losses and dispersion, especially in thin films, represent singular perturbations that qualitatively alter the spectral content and the properties of eigenwaves. To address these problem, eigenmodes in lossy metallic and semiconductor films and waveguides have been studied numerically by directly solving the rigorous dispersion equations - see, e.g., [14]-[20]. Even though the latter approach has enabled the analysis of SPP modes in certain structures, it frequently suffers from uncertainty in identification of the physical solutions and interpretation of the numerical results [10]. Besides, the effects of loss and dispersion of permittivity on the other types of eigenwaves, such as complex, leaky [6], [7] and dynamic (bulk) waves, is scantly addressed in the literature.

In this paper, the complete spectrum and properties of TM eigenwaves in the canonical layered structures containing semiconductor and $\mathrm{Au}$ thin films have been investigated in a broad frequency range using the rigorous solutions of the full-wave dispersion equation (DE), and the field and Poynting vector distributions. The model and generic properties of the DE are discussed in Section 2. In Section 3, the asymptotic solutions of the DE are obtained for the SPP and complex waves in lossy films, and attenuation of the SPP is assessed. The effects of the structure parameters on the properties of eigenwaves in the lossy semiconductor films are discussed in Section 4 based upon a comprehensive numerical analysis. In Section 5, the features of SPP in Au film with the optical permittivity given by table data [21] are discussed in detail. The major properties of the SPP modes in lossy layered structures are summarised in Section 6.

\section{The model and problem statement}

The eigenwave problems for the layered wave guiding structures lead to the cumbersome transcendental equations, which are usually treated numerically with little control over the completeness and physical meaning of the obtained solutions. These difficulties are further escalated when losses and frequency dispersion of the material parameters should be taken into account in a rigorous manner. On the other hand, the main properties of eigenwaves in complex layered arrangements can be inferred from the simplified canonical models that adequately describe the fundamental features of the original physical structures. The latter approach has been adopted in this paper to examine the spectrum and properties of eigenmodes guided by the lossy layered structures containing semiconductor and metallic films.

Let us consider the canonical 3-layer planar structure in Fig. 1 composed of a semiconductor or metallic film with relative permittivity $\varepsilon_{s}(\omega)$ and thickness

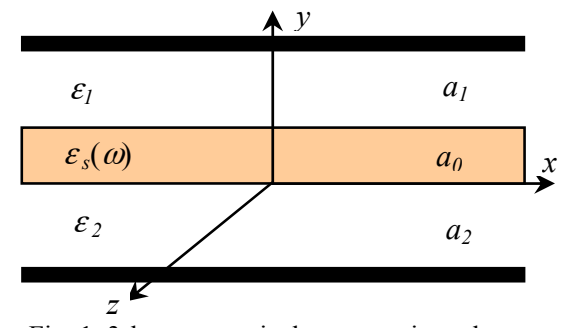

Fig. 1. 3-layer canonical structure in a planar waveguide with perfect conductor walls. 
$a_{0}$ sandwiched between two dielectric layers with relative permittivities $\varepsilon_{1,2}$ and thicknesses $a_{1,2}$, all enclosed into a parallel-plate waveguide with perfect conductor walls ${ }^{1}$. The film permittivity $\varepsilon_{s}(\omega)$ is either described by Drude model (1) or given by the table data for metals or semiconductor plasma

$$
\varepsilon_{s}(\omega)=\varepsilon_{L}\left[1-\omega_{p}^{2} / \omega(\omega-i v)\right]
$$

where $\omega$ is angular frequency and the time convention is assumed in the form $\exp (i \omega t) ; \varepsilon_{L}$ is relative background permittivity; $\omega_{p}$ and $v$ are the plasma and collision frequencies which generally may be slowly varying functions of $\omega$, e.g., when $\varepsilon_{s}(\omega)$ is given by the table data.

The complete spectrum of eigenwaves in the 3-layer structure of Fig. 1 comprises two orthogonal sets of eigenwaves, TM and TE (alternatively called LSE and LSM), which can be treated independently [22]. The TE waves represent ordinary waves in a planar dielectric waveguide, which are the bound modes of the central layer only at frequencies above the cut-off at $\operatorname{Re} \varepsilon_{s}(\omega)>1$. Since these cut-off frequencies are determined by the layer thickness which must be commensurate with the wavelength, the TE waves are guided by thin films only at very high frequencies $\omega>>\omega_{p}$. Conversely, the fundamental TM waves can propagate at any frequency and their spectrum comprises not only the bulk dynamic waves but also the SPP modes that are of primary interest in this work. Therefore only TM waves with the field components $H_{x}, E_{y}$ and $E_{z}$ travelling along $z$-axis will be considered in the remainder of this paper.

Enforcing the boundary conditions of tangential field continuity at the layer interfaces, the dispersion equation (DE) for TM waves can be expressed in the following form

$$
\left(\beta_{0 n}^{2}+A_{1} A_{2} \varepsilon_{s}^{2}\right) \tanh \left(\beta_{0 n} k_{0} a_{0}\right)+\beta_{0 n}\left(A_{1}+A_{2}\right) \varepsilon_{s}=0
$$

where the time $t$ and $z$-coordinate dependences are assumed in the form $\exp \left\{i\left(\omega t-k_{0} \gamma_{n} z\right)\right\}$; $k_{0}$ is the free space wavenumber and $\gamma_{n}$ is the normalised longitudinal wavenumber;

$$
\beta_{0 n}=\sqrt{\gamma_{n}^{2}-\varepsilon_{s}(\omega)} ; \quad \beta_{m n}=\sqrt{\gamma_{n}^{2}-\varepsilon_{m}} ; \quad A_{m}=\frac{\beta_{m n} \tanh \left(\beta_{m n} k_{0} a_{m}\right)}{\varepsilon_{m}} ; \quad m=1,2 .
$$

Simplicity and analyticity of the DE have been perceived as the essential prerequisites of the model used. The DE (2) meets these requirements and is amenable to the analytical treatment which provides a physical insight in the features of eigenmode spectrum and interrelation between the SPP attenuation and losses in the semiconductor and metallic films. This model additionally provides the necessary flexibility for evaluating the effects of the extrinsic parameters, such as dielectric layer permittivities $\varepsilon_{1,2}$ and thicknesses $a_{1,2}$, on the eigenwaves in the asymmetric arrangements. Furthermore the closed form asymptotic expansions of the DE (2) for several limiting cases facilitate the traceable numerical solutions and consistent interpretation of the simulation results.

\section{Asymptotic analysis of the dispersion equation}

The basic properties of the eigenwave spectrum and, particularly, SPP can be inferred directly from the DE (2). At frequencies $\omega<\omega_{p}$, where $\operatorname{Re} \varepsilon_{s}(\omega)<0$, the DE has the solutions at $\operatorname{Re} \gamma_{n}>>1$ which can be obtained by the asymptotic expansion of (2). In order to simplify the asymptotic treatment, it is convenient to rearrange the DE (2) into the factorised form, using the explicit expression (1) for $\varepsilon_{s}(\omega)$

$$
\left[\omega-\omega_{1}\left(\gamma_{n}, \omega\right)\right]\left[\omega-\omega_{2}\left(\gamma_{n}, \omega\right)\right]=0
$$

where

$$
\omega_{m}\left(\gamma_{n}, \omega\right)=\frac{\omega_{p}}{\operatorname{Re} \sqrt{1+\frac{\beta_{0 n} \operatorname{coth}\left(\beta_{0 n} k_{0} a_{0}\right)}{2 A_{1} A_{2}}\left[A_{1}+A_{2}+(-1)^{m} \sqrt{\left(A_{1}-A_{2}\right)^{2}+\frac{4 A_{1} A_{2}}{\cosh ^{2}\left(\beta_{0 n} k_{0} a_{0}\right)}}\right.}}, \quad m=1,2 .
$$

The principal solutions of (3) at $\operatorname{Re} \gamma_{n}>>1$ are readily recognisable as the SPP modes guided by the central layer, cf. [1], [2], [10]. Indeed, taking into account that the frequency dependence of $\omega_{m}\left(\gamma_{n}, \omega\right)$ is contained only in the terms $k_{0} a_{0}$ and $\left|\cosh ^{2}\left(\beta_{0 n} k_{0} a_{0}\right)\right| \rightarrow 0$ at $\operatorname{Re} \gamma_{n} \gg 1 / k_{0} a_{0}, \omega_{m}\left(\gamma_{n}, \omega\right)$ given by (4) can be further simplified as follows:

$$
\omega_{m}\left(\gamma_{n}, \omega\right)=\omega_{p} \operatorname{Re} \sqrt{\frac{\varepsilon_{L} \beta_{m n}}{\varepsilon_{L} \beta_{m n}+\varepsilon_{m} \beta_{0 n}}}+O\left(e^{-2 \operatorname{Re} \beta_{m n} k_{0} a_{m}}\right)+O\left(e^{-2 \operatorname{Re} \beta_{0 n} k_{0} a_{0}}\right), \quad m=1,2
$$

where at $\operatorname{Re} \beta_{0 n} \rightarrow \infty, \omega_{m}\left(\gamma_{n}, \omega\right)$ tends to the frequencies $\omega_{a s 1,2}$ of the plasmonic resonances at the respective interfaces [1], [2]. For further reference, these frequencies are convenient to represent in the normalised form scaled to $\omega_{p}$

$$
\omega_{\text {nas } 1,2}=\frac{\omega_{a s 1,2}}{\omega_{p}}=\sqrt{\frac{\varepsilon_{L}}{\varepsilon_{L}+\varepsilon_{1,2}}},
$$

\footnotetext{
${ }^{1}$ The waveguide enclosure is used here with a sole purpose of formulating the boundary value problem for the bounded structure that enables us to represent the dispersion equation as an analytical function without branch cuts, cf. (2). The latter feature is essentially exploited for the rigorous computations of the complete spectrum of eigenwaves including complex and leaky waves.
} 
The asymptotic form (5) of the SPP dispersion characteristics has been obtained under the assumption of $\operatorname{Re} \gamma_{n} \gg 1 / k_{0} a_{0}$. The latter requirement is exceedingly constrictive for the thin film structures, and validity of the basic asymptotic solutions (5), (6) is further limited in presence of losses. Indeed, examination of (4) shows that at $k_{0} a_{0}<<1$, the last term in (4), which describes the field interactions between the film interfaces, is sufficiently small only at very large values of $\left|\beta_{0 n}\right|>>1 / k_{0} a_{0}$. However the required values of $\operatorname{Re} \beta_{0 n}$ may be unattainable in actual metallic and semiconductor films because of SPP attenuation. Also, the contribution of this exponentially small term is no negligible at $A_{1} \approx A_{2}$, and even may become dominant at any thicknesses of the external dielectric layers. For example at $\varepsilon_{1}=\varepsilon_{2}$, the plasmonic resonance frequencies $\omega_{a s 1,2}$ are degenerated, cf. (6), but the even and odd SPP modes differ and their dispersion characteristics do not intersect each other at any finite $\operatorname{Re} \beta_{0 n}$ as illustrated by the results of numerical analysis in Section 4. Also, it is necessary to note that the full geometrical symmetry of the structure is no a prerequisite for existence of the even and odd SPP modes as long as the condition of $A_{1}=A_{2}$ is satisfied. This implies that the field symmetry inside the film can be realised with dissimilar dielectric layers as their parameters fulfil the condition $A_{1}=A_{2}$.

It is important to emphasise that the conventional asymptotic representation (5) of the SPP dispersion characteristics is valid only for the lossless structures. When loss is taken into account, $\gamma_{n}$ becomes complex valued quantity and the DE requires more delicate treatment to evaluate not only the propagation constant but also the attenuation constant. Making use of the relationship between $\beta_{0 n}$ and $\beta_{m n}$, and separating the real and imaginary parts in the DE (2), an asymptotic expansion for the attenuation constant of SPP at $v<<\omega$ can be represented in the following form

$$
\operatorname{Im} \gamma_{n}(\omega) \simeq \frac{v \omega\left(\operatorname{Re} \gamma_{n}(\omega)\right)^{3}}{2 \omega_{p} \operatorname{Re}\left(\varepsilon_{m}+\left|\varepsilon_{s}(\omega)\right|\right)}\left[1+O\left(\left[\frac{\operatorname{Im} \gamma_{n}(\omega)}{\operatorname{Re} \gamma_{n}(\omega)}\right]^{2}\right)\right], \quad m=1,2
$$

The latter expression reveals an important property of the SPP attenuation, which rapidly grows with $\operatorname{Re} \gamma_{n}$ at the unusually high rate of $\sim\left(\operatorname{Re} \gamma_{n}\right)^{3}$. This feature of the SPP is in sharp contrast to any kind of dynamic waves whose attenuation constants increase linearly with $\operatorname{Re} \gamma_{n}$. Thus the SPP modes inherently experience much stronger attenuation even in the low loss media with small $v$. Such a kind of the attenuation behaviour described by (7) is not unique to the SPPs, but also was earlier reported in [23] for the surface magnetostatic waves. This phenomenon is intimately related to the counter-directed power flows (Poynting vectors) in the semiconductor/metal film with $\operatorname{Re} \varepsilon_{\mathrm{s}}(\omega)<0$ and surrounding dielectric layers as illustrated by the results of numerical simulations in [28] and also discussed in Sections 4, 5. Finally, it is important to note that while the asymptotic formula (7) provides a useful qualitative estimate of the SPP decay, its applicability is restricted to the cases of low loss $(\nu<<\omega)$ and weak attenuation $\operatorname{Im} \gamma_{n}<\operatorname{Re} \gamma_{n}$.

As already mentioned earlier, the conventional asymptotic analysis of the DE (2) may become invalid for thin lossy films when $k_{0} a_{0}<<1$. In this case, the DE (2) can alternatively be represented in the following form

$$
\beta_{0 n}=\frac{1}{2 k_{0} a_{0}}\left[\ln \frac{\left(\beta_{0 n}-A_{1 n} \varepsilon_{s}\right)\left(\beta_{0 n}-A_{2 n} \varepsilon_{s}\right)}{\left(\beta_{0 n}+A_{1 n} \varepsilon_{s}\right)\left(\beta_{0 n}+A_{2 n} \varepsilon_{s}\right)}+i m \pi\right] ; \quad m=0, \pm 1, \ldots .
$$

Comparison of (2) and (8) shows that while the fundamental SPP modes are the principal solutions of the latter equation at $m=0$, an infinite countable set of complex modes co-exists even in the lossless structure. Similar property of the eigenmode spectrum is known for the magnetostatic waves existing in the frequency range of negative effective permeability of thin YIG films [24], [25]. An important implication of (8) is that in presence of loss, the complex waves may become the dominant modes and exhibit lower attenuation than the "loss perturbed" fundamental modes of the lossless structure. This feature is important for the qualitative understanding of the properties of complete eigenmode spectrum, mode identification and interpretation of the simulation results presented in following Sections.

\section{Full-wave analysis of eigenmodes in lossy semiconductor-dielectric structures}

In order to investigate the full spectrum and properties of the eigenwaves in thin films, the DE (2) has been treated numerically in the range of frequencies and the structure parameters. This problem poses considerable computational challenges associated with the multi-scale geometry and the spectrum singularities due to the plasmonic resonances. In the case of lossy structures the difficulties are compounded by the fact that the wavenumbers are complex valued quantities corresponding to the complex roots of the complex valued DE. Furthermore the eigenmode identification necessitates that all the roots contained in the specified domains of the complex plane of wavenumbers be determined.

Evaluation of complex roots of complex valued functions is a notoriously hard problem which is fraught with the difficulties of counting, locating, bracketing and refining the solutions. Nevertheless for certain classes of analytical functions, their roots can be reliably calculated with the aid of the principle of argument combined with the gradient methods [26], [27]. Since the DE (2) for the bounded layered structure can be cast in the form of an analytical function, the latter approach has been adopted here for the analysis of eigenwaves in layered structure of Fig. 1.

The numerical simulations of the SPP characteristics have been facilitated by the asymptotic expressions (7), (8) which provide the initial approximations to the sought eigenvalues. Then owing to the DE analyticity, the wavenumbers $\gamma_{n}(\omega)$ have been progressively calculated by tracking $\gamma_{n}(\omega)$ dependencies and using the already obtained $\gamma_{n}$ values for initialisation of the search procedure at the next frequency point. This approach has enabled a highly efficient and robust algorithm for the analysis and identification of the eigenmodes across a broad frequency range. 
The spectrum and characteristics of the eigenmodes including SPP, dynamic (bulk) and complex waves in the canonical 3-layer structure containing lossy semiconductor films (Fig. 1) have been obtained by the numerical solution of the DE (2) and are examined in this Section. Starting from the lossless and symmetric arrangements, the effects of loss in semiconductor film, and thicknesses and permittivities of dielectric layers are discussed in detail. The following parameters of $n$-GaAs semiconductor film are assumed unless specified otherwise: $\varepsilon_{L}=13.1, \omega_{p}=848.17 \times 10^{9} \mathrm{rad} / \mathrm{sec}$, and $v=0.01 \omega_{p}$. To illustrate the generic features of the eigenmodes independently of the particular material parameters, all frequencies are scaled to $\omega_{p}$, i.e. $\omega_{n}=\omega / \omega_{p}$ is the normalised frequency and $v_{n}=v / \omega_{p}$.

\subsection{SPP in the lossless structure with semiconductor film}

In order to consistently assess the effect of loss on the waves in the semiconductor film with the Drude permittivity $\varepsilon_{s}(\omega)$ defined in (1), let us first review the major features of the waves in respective lossless structure $\left(v_{n}=0\right)$. The eigenmode spectrum at $\omega_{n}<1$ includes the SPP modes and complex waves. The dispersion characteristics $\gamma_{n}\left(\omega_{n}\right)$ of the fundamental SPP modes are shown in Fig. 2 for the symmetric structure with $\varepsilon_{1}=\varepsilon_{2}=3.9$ and an asymmetric arrangement containing dielectric layers with dissimilar permittivities: $\varepsilon_{1}=9, \varepsilon_{2}=3.9$.

Two fundamental SPP modes shown in Fig. 2 are usually referred to as a "short-range" $\left(\mathrm{O}^{+}\right)$and "long-range" $\left(\mathrm{C}^{\prime}\right.$ and $\mathrm{O}^{-}$) SPP [5]. Their main properties in the symmetric structure of Fig. 1 can be summarised as follows:

- The modes $\mathrm{C}^{\prime}$ and $\mathrm{O}^{-}$represent a pair of "long-range" SPP modes in the semiconductor film.

- All three SPP modes $\mathrm{O}^{+}, \mathrm{C}^{\prime}$ and $\mathrm{O}^{-}$are the bound waves, and their fields exponentially decay away from the film surfaces.

- The cross-sectional distributions of longitudinal electric field $E_{z}\left(y-a_{0} / 2\right)$ have an even symmetry in the mode $\mathrm{O}^{+}$and an odd symmetry in the modes $\mathrm{C}^{\prime}$ and $\mathrm{O}^{-}$.

- The SPP modes $\mathrm{O}^{+}$and $\mathrm{C}^{\prime}$ are forward waves, while the mode $\mathrm{O}^{-}$is a backward wave; the modes $\mathrm{O}^{+}$and $\mathrm{O}^{-}$ experience cut-off at the plasmonic resonance frequency $\omega_{\text {nas }}=\omega_{\text {nas } 1}=\omega_{\text {nas } 2}$ defined by eq. (6).

- In thin films, the SPP mode $\mathrm{C}^{\prime}$ expands into the bandgap $\omega_{\text {nas }}<\omega_{n}<1$, where it coexists with the SPP mode $\mathrm{O}^{-}$which has $\gamma_{n}>\gamma_{n c}>\sqrt{\max \left(\varepsilon_{1}, \varepsilon_{2}, \varepsilon_{L}\right)}$; the dispersion curves of the modes $\mathrm{C}^{\prime}$ and $\mathrm{O}^{-}$merge at their common cut-off frequency $\omega_{n c}=\omega_{n}\left(\gamma_{n c}\right)>\omega_{n a s}$, where $d \omega_{n} /\left.d \gamma_{n}\right|_{\gamma_{n}=\gamma_{n c}}=0$, see Fig. 2 .

In the asymmetric structure with dissimilar dielectrics $\left(\varepsilon_{1}>\varepsilon_{2}\right)$, the SPP spectrum content differs and the SPP modes exhibit the additional features, illustrated in Fig. 2, cf. [3], [6].

- The plasmonic resonance frequencies associated with the modes $\mathrm{O}^{+}$and $\mathrm{O}^{-}$differ and equal to $\omega_{\text {nasl }}$ and $\omega_{\text {nas }}$ $\left(\omega_{\text {nas } 1}<\omega_{\text {nas }}=\omega_{\text {nas } 2}\right)$, respectively, cf. (6).

- At low frequencies $\omega_{n}<\omega_{n b}\left(\omega_{n b}\right.$ is defined by the relation $\gamma_{n}\left(\omega_{n b}\right)=\sqrt{\varepsilon_{1}}$, see insert in Fig. 2), the SPP mode $\mathrm{C}^{\prime}$ is a leaky wave, radiating into a dielectric layer with the higher permittivity $\varepsilon_{1}$, cf. [7]. The mode $\mathrm{C}^{\prime}$ becomes a bound wave at $\omega_{n b}<\omega_{n}<\omega_{n c}$. $\omega_{n b}$ increases in thinner films, so that the mode $\mathrm{C}^{\prime}$ may become a bound wave only in the bandgap, as $\omega_{n}>\omega_{n b}>\omega_{n a s}$. In the latter case, its band $\omega_{n b}<\omega_{n}<\omega_{n c}$ shrinks and becomes narrower than for the backward SPP mode $\mathrm{O}^{-}\left(\omega_{\text {nas }}<\omega_{n}<\omega_{n c}\right)$.

When the structure asymmetry is further increased by the substantially dissimilar thicknesses of the dielectric layers, the SPP spectrum and properties qualitatively alter [28]. This effect is more pronounced when the dielectric layer thickness is

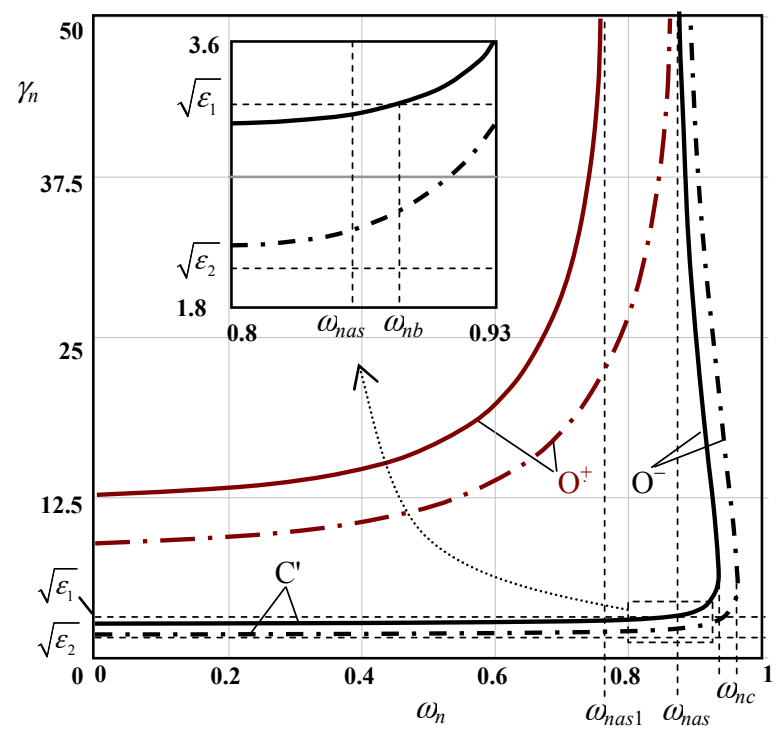

Fig. 2. Dispersion diagrams $\gamma_{n}\left(\omega_{n}\right)$ of SPP in the lossless $\left(v_{n}=0\right)$ symmetric (dash-dot lines, $\varepsilon_{1}=\varepsilon_{2}=3.9$ ) and asymmetric (solid lines, $\left.\varepsilon_{1}=9, \varepsilon_{2}=3.9\right)$ structures with the parameters: $a_{0}=20 \mu \mathrm{m}$; $a_{1}=a_{2}=40 \mu \mathrm{m}, \varepsilon_{L}=13.1, \omega_{p}=848.17 \times 10^{9} \mathrm{rad} / \mathrm{sec}$. The curves $\mathrm{O}^{-}$ and $\mathrm{C}^{\prime}$ are associated with long-range SPP and $\mathrm{O}^{+}$- with short-range SPP. Insert shows a magnified plot about the frequency $\omega_{n b}$ where a leaky SPP mode $\mathrm{C}^{\prime}$ in the asymmetric structure with $\varepsilon_{1}>\varepsilon_{2}$ turns into a bound mode. Note that a pair of the bound long-range SPP, $\mathrm{O}^{-}$and $\mathrm{C}^{\prime}$, exists in the frequency band $\omega_{\text {nas }}<\omega_{n}<\omega_{n c}$

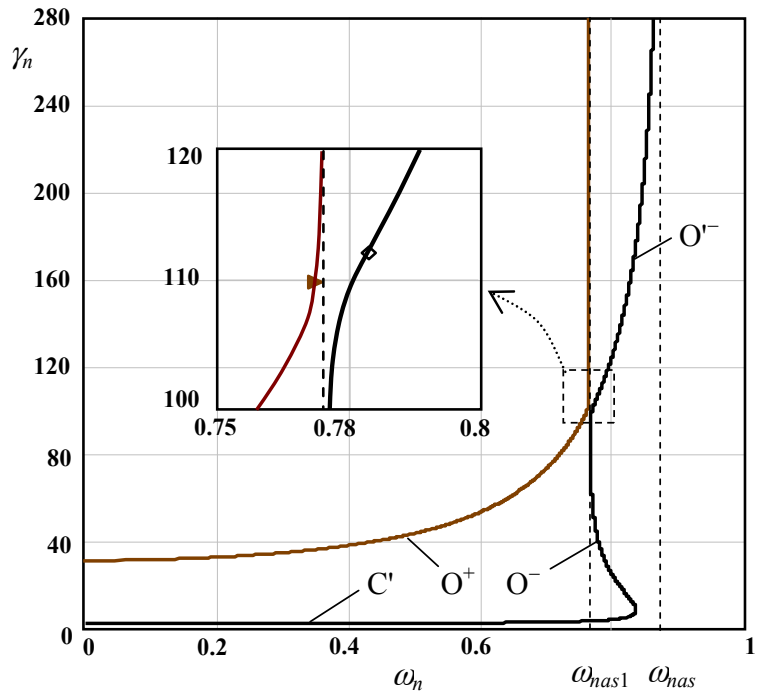

Fig. 3. Dispersion diagram $\gamma_{n}\left(\omega_{n}\right)$ of SPP in the lossless $\left(v_{n}=0\right)$ asymmetric structure with dissimilar dielectric layers. The structure parameters: $a_{0}=20 \mu \mathrm{m} ; \varepsilon_{L}=13.1, a_{1}=40 \mu \mathrm{m}, a_{2}=2 \mu \mathrm{m}, \varepsilon_{1}=9$, $\varepsilon_{2}=3.9, \quad \omega_{p}=848.17 \times 10^{9} \mathrm{rad} / \mathrm{sec}$. The curves $\mathrm{O}^{-}$and $\mathrm{C}^{\prime}$ are associated with the long-range SPP and $\mathrm{O}^{+}$with the short-range SPP. Insert shows a magnified plot near the "collision" point of the modes $\mathrm{O}^{+}, \mathrm{O}^{-}$and $\mathrm{O}^{-}$at frequency $\omega_{\text {nas } 1}$. 
commensurable with the scale of the SPP confinement to the film interfaces. In particular, variations of $a_{2}$ (a layer with lower permittivity) cause significant distortion of both modes $\mathrm{O}^{-}$and $\mathrm{O}^{+}$and lead to appearance of an additional SPP mode $\mathrm{O}^{-}$as illustrated in Fig. 3. Namely, when $a_{2}$ decreases, the mode $\mathrm{O}^{+}$rapidly approaches the plasmonic resonance at $\omega_{n}=\omega_{\text {nas } 1}-0$. At the same time, the backward mode $\mathrm{O}^{-}$migrates into the frequency band $\omega_{\text {nas } 1}<\omega_{n}<\omega_{\text {nas }}$, "collides" with the mode $\mathrm{O}^{+}$and turns into the forward SPP mode $\mathrm{O}^{-}$. This phenomenon occurs only when the dielectric layer of lower permittivity is very thin and the mode $\mathrm{O}^{-}$is strongly distorted. Analysis of the cross-sectional field and Poynting vector distributions in [28] has shown that in vicinity of the "collision" point, the modes $\mathrm{O}^{+}$and $\mathrm{O}^{-}$interchange their locations at the semiconductor film interfaces and the forward mode $\mathrm{O}^{\prime-}$ is confined to the same interface as the mode $\mathrm{O}^{+}$. Although the dispersion curve of the mode $\mathrm{O}^{-}$looks like an extension of the mode $\mathrm{O}^{+}$into the frequency band $\omega_{\text {nas } 1}<\omega_{n}<\omega_{\text {nas }}$, the parity ${ }^{2}$ of the field distribution in the mode $\mathrm{O}^{-}$remains the same as in the mode $\mathrm{O}^{-}$, i.e. $E_{z}$ component of both modes $\mathrm{O}^{-}$and $\mathrm{O}^{-}$has a null inside of the film.

The outlined features of the SPP in the lossless semiconductor films provide the basis for the comparative analysis of the effect of loss on the eigenmode spectrum and properties discussed in the following Sections.

\subsection{Eigenmodes in the symmetric structure with lossy semiconductor film}

The normalised propagation $\left(\operatorname{Re} \gamma_{n}\right)$ and attenuation $\left(\operatorname{Im} \gamma_{n}\right)$ constants of the fundamental eigenmodes in the symmetric structure with lossy $\left(v_{n}=0.01\right)$ and lossless $\left(v_{n}=0\right)$ semiconductor films are shown in Fig. 4 for the same parameters as in Fig. $2\left(a_{0}=20 \mu \mathrm{m}, a_{1}=a_{2}=40 \mu \mathrm{m}, \varepsilon_{1}=\varepsilon_{2}=3.9\right)$. In presence of loss, the dispersion curves $\operatorname{Re} \gamma_{n}\left(\omega_{n}\right)$ for the SPP modes $\mathrm{A}, \mathrm{B}$ and $\mathrm{D}$, and the dynamic mode $\mathrm{C}$ closely follow the respective curves for the modes $\mathrm{O}^{+}, \mathrm{C}^{\prime}, \mathrm{O}^{-}$and $\mathrm{C}$ in the lossless structure (the label $\mathrm{C}$ is used in both lossless and lossy cases). Inspection of the plots for $\operatorname{Im} \gamma_{n}$, however, exposes the qualitative differences between the modes $\mathrm{D}$ and $\mathrm{O}^{-}$existing in the frequency band $\omega_{\text {nas }}<\omega_{n}<1$, where the mode $\mathrm{D}$ exhibits strong attenuation in presence of small losses. To elucidate the effect of loss on the SPP properties, it is necessary to examine the eigenmodes at the plasmonic resonance $\omega_{\text {nas }}$ in more detail.

When the mode A approaches $\omega_{\text {nas }}$, its attenuation constant, $\operatorname{Im} \gamma_{n}$, grows much faster than $\operatorname{Re} \gamma_{n}$ as predicted by the asymptotic estimate in (7). Then as $\operatorname{Re} \gamma_{n}$ reaches its peak at $\omega_{n} \approx \omega_{\text {nas }}$, the propagating mode $\mathrm{A}$ turns into a backward complex mode $\mathrm{D}$. The distinctive feature of the mode $\mathrm{D}$ is that its $\operatorname{Re} \gamma_{n}$ is nearly the same as in the mode $\mathrm{O}^{-}$, but $\operatorname{Im} \gamma_{n}$ is huge, i.e. the mode $\mathrm{D}$ represents the loss perturbed complex wave of the lossless structure, cf. (8). Thus at $\omega_{n}>\omega_{\text {nas }}$, a low loss counterpart of the backward long-range SPP mode $\mathrm{O}^{-}$does not exist in the lossy thin film.

The SPP mode B represents the loss perturbed long-range SPP mode $\mathrm{C}^{\prime}$. It experiences a resonance at the cut-off frequency $\omega_{n}=\omega_{n c}$ of the mode $\mathrm{C}^{\prime}$, where it converts into a complex mode $\mathrm{F}$ of the planar dielectric waveguide. It is important to note that unlike to the modes $\mathrm{C}^{\prime}$ and $\mathrm{O}^{-}$, the modes $\mathrm{B}$ and $\mathrm{D}$ are disjointed at $\omega_{n}=\omega_{n c}$ and evolve into the complex modes with substantially different attenuation constants as illustrated in Fig. 4. Thus the small losses in the semiconductor film inflict the qualitative changes of the eigenmode spectrum of the respective lossless structure.

To gain a deeper insight into the mechanisms of SPP propagation in semiconductor films, field and Poynting vector $(\boldsymbol{P})$ distributions of the SPP modes have been examined. While both the modes $\mathrm{A}$ and $\mathrm{B}$ are the bound eigenwaves, the patterns of their longitudinal power flow $\left(\operatorname{Re} P_{z}\right)$ substantially differ. Namely, $\operatorname{Re} P_{z}$ of the SPP mode A is tighter confined to the film interfaces and has a null inside the film, whereas $\operatorname{Re} P_{z}$ of the
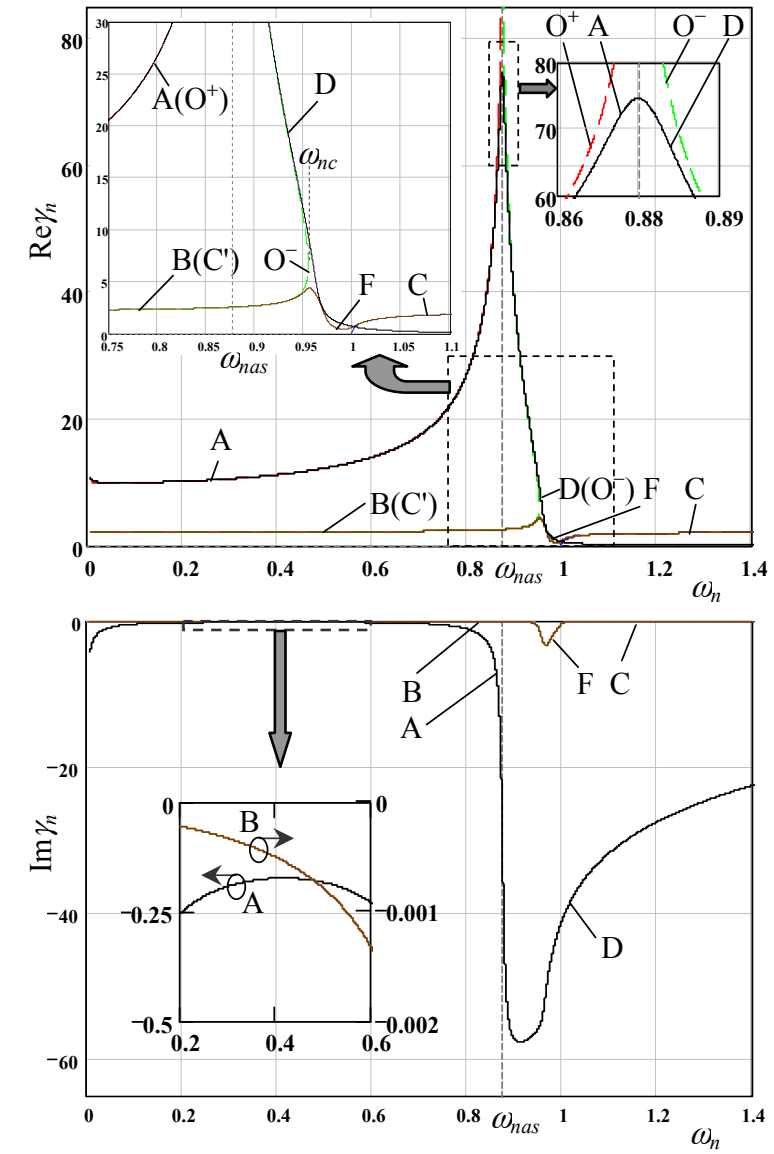

Fig. 4. Propagation $\left(\operatorname{Re} \gamma_{n}\right)$ and attenuation $\left(\operatorname{Im} \gamma_{n}\right)$ constants of the lowest eigenmodes in the lossless and lossy symmetric structures with the parameters: $\varepsilon_{1}=\varepsilon_{2}=3.9, a_{1}=a_{2}=40 \mu \mathrm{m}$, and $a_{0}=20 \mu \mathrm{m}$; $\varepsilon_{L}=13.1, \omega_{p}=848.17 \times 10^{9} \mathrm{rad} / \mathrm{sec}$. The SPP modes A and B, complex mode $\mathrm{D}$ and dynamic mode $\mathrm{C}$ in the lossy film with $v_{n}=0.01$ are juxtaposed with respective modes $\mathrm{O}^{+}, \mathrm{O}^{-}, \mathrm{C}^{\prime}$ and $\mathrm{C}$ in the lossless structure $\left(v_{n}=0\right)$. The curves for $\operatorname{Re} \gamma_{n}$ of the modes $\mathrm{A}$ and $\mathrm{O}^{+}$overlap at $\omega_{n}<\omega_{n a s}, \mathrm{D}$ and $\mathrm{O}^{-}$overlap at $\omega_{n a s}<\omega_{n}<\omega_{n c}$, B and $\mathrm{C}^{\prime}$ overlap at $\omega_{n}<\omega_{n c}$.

\footnotetext{
${ }^{2}$ Despite the field symmetry of the modes $\mathrm{O}^{-}$and $\mathrm{O}^{+}$is significantly distorted in strongly asymmetric structures, the respective fields retain the fundamental notions of the original odd and even symmetry. For example, $E_{z}$ component of the mode $\mathrm{O}^{-}$has a null inside of the film, while the mode $\mathrm{O}^{+}$does not have it.
} 
SPP mode B is spread over the entire film thickness and slowly decays in the surrounding dielectric layers [28]. On the other hand, Poynting vectors of both the modes A and B demonstrate the following important common traits:

- the longitudinal components, $\operatorname{Re} P_{z}$, inside and outside of the semiconductor film are counter-directed;

- the transverse components, $\operatorname{Re} P_{y}$, point into the film at both interfaces;

- curls of electric field are formed about the film interfaces, cf. Fig. 7 in [8].

Taking into account these features and the fact that Poynting vector represents the local rate of energy transport at any point [29], these patterns of Poynting vector distributions in SPP can be attributed to vortices of power flux, cf. Fig. 1c in [4], which trap a portion of the total power carried by SPP. Such a description of the SPP energy transport provides a consistent interpretation of the SPP properties and the characteristics shown in Fig. 4. Indeed, at frequencies close to the plasmonic resonance $\omega_{n} \rightarrow \omega_{\text {nas }}-0$, the total power flow in SPP modes vanishes, while $\operatorname{Re} \gamma_{n}$ grows and Poynting vectors remain finite at any point of the structure cross-section. Therefore the plasmonic resonance and SPP cut-off at $\omega_{\text {nas }}$ are caused not by decay of the local power flow but by its vorticity, where the net power transported in opposite directions in the semiconductor film and in surrounding it dielectric layers become equal. Thus the dispersion and attenuation properties of the SPP modes can be unambiguously correlated with the vorticity of the SPP Poynting vectors. For example, the higher vorticity of the power flow in the mode A than in the mode B manifests itself in the slower propagation (larger $\operatorname{Re} \gamma_{n}$ ) and higher attenuation, see Fig. 4. These observations also suggest that the vorticity of power flow is responsible for the higher attenuation rate of SPP $\left(\operatorname{Im} \gamma_{n} \sim v\left(\operatorname{Re} \gamma_{n}\right)^{3}\right)$ than the TM modes propagated in dielectric waveguides.

The modes $\mathrm{D}$ and $\mathrm{F}$, existing in the frequency band $\omega_{\text {nas }}<\omega_{n}<\sqrt{1-v_{n}^{2}}$, are complex leaky waves whose power flows are no longer confined to the film interfaces but radiate into exterior dielectric layers. It is necessary to note that despite the dispersion characteristics of the complex modes $\mathrm{D}$ and $\mathrm{F}$ have negative slope $\left(d \omega_{n} / d \operatorname{Re} \gamma_{n}<0\right)$ usually associated with the backward wave type, their total power flows over the whole waveguide cross-section remains co-directional with the wave vector for both modes. Thus the group velocity direction of the strongly attenuated complex modes $\mathrm{D}$ and $\mathrm{F}$ inferred from their dispersion curves is no representative of their power flow, cf. [30].

\subsection{Eigenmodes in asymmetric structure}

In previous Section, it was shown that losses in the semiconductor film inflict the qualitative changes in the spectrum and properties of the eigenwaves in the respective lossless structure. The effects of the structure asymmetry such as dissimilar permittivities and thicknesses of dielectric layers on the properties of eigenwaves in the lossy semiconductor film are discussed next in comparison with the respective lossless cases considered in Section 4.1.

\subsubsection{Dielectric layers with dissimilar permittivities $\left(\varepsilon_{1} \neq \varepsilon_{2}\right)$.}

Let us consider first a symmetric geometry with dissimilar dielectric layers with permittivities $\varepsilon_{1} \neq \varepsilon_{2}$ ( $\varepsilon_{1}=9, \quad \varepsilon_{2}=3.9$ ) and the other parameters unchanged: $\varepsilon_{L}=13.1, a_{0}=20 \mu \mathrm{m}, a_{1}=a_{2}=40 \mu \mathrm{m}$, and $\omega_{p}=848.17 \times 10^{9}$ $\mathrm{rad} / \mathrm{sec}$.

Unequal permittivities $\varepsilon_{1,2}$ of dielectrics surrounding the semiconductor film distort the symmetry of the field patterns in the waveguide cross-section that entails the changes in the spectrum and properties of eigenwaves, cf. Fig. 2 and Fig. 5. Since the plasmonic resonances $\omega_{\text {nas } 1,2}$ defined by eq. (6) are split in the asymmetric structure, a bandgap arises at $\omega_{\text {nas } 1}<\omega_{n}<\omega_{\text {nas } 2}=\omega_{\text {nas }}$ where only the long-range SPP mode $C^{\prime}$ and non-propagating evanescent waves exist in the lossless case, see Fig. 2. In presence of loss, the evanescent modes turn into the strongly attenuated complex waves, e.g., mode G shown in Fig. 5.

At frequencies $\omega_{n}<\omega_{\text {nas } 1}$, the eigenmodes $\mathrm{A}$ and $\mathrm{B}$ correspond to the loss perturbed modes $\mathrm{O}^{+}$and $\mathrm{C}^{\prime}$ (see Fig. 2). The SPP mode $B$ is a bound wave of the semiconductor film only when $\omega_{n}$ approaches the
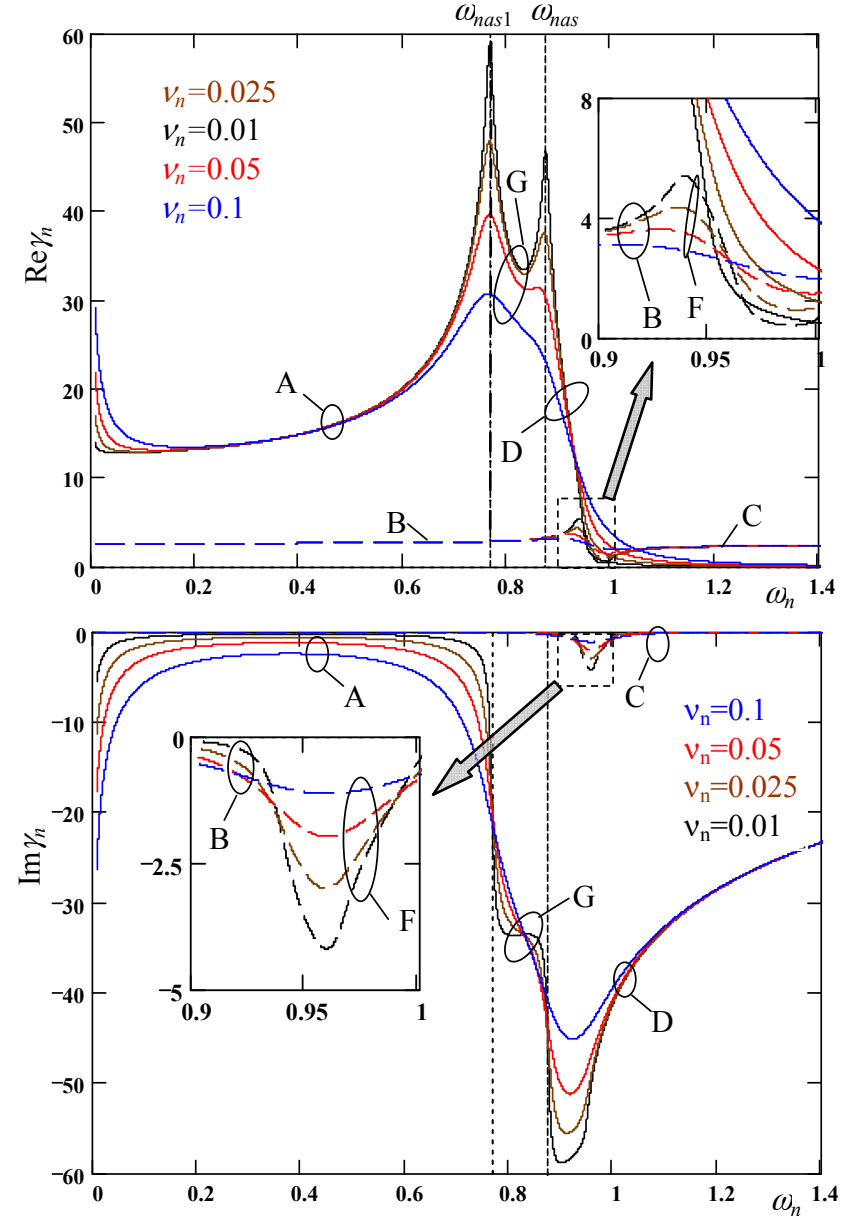

Fig. 5. Propagation $\left(\operatorname{Re} \gamma_{n}\right)$ and attenuation $\left(\operatorname{Im} \gamma_{n}\right)$ constants of eigenmodes in the asymmetric structure vs. collision frequency $v_{n}=0.01,0.025,0.05$ and 0.1. The structure parameters: $\varepsilon_{1}=9, \varepsilon_{2}=3.9, \varepsilon_{L}=13.1, \omega_{p}=848.17 \times 10^{9}$ $\mathrm{rad} / \mathrm{sec}, a_{0}=20 \mu \mathrm{m}, a_{1}=a_{2}=40 \mu \mathrm{m}$. Inserts show the enlarged plots near the resonance of the SPP mode $B$. 
plasmonic resonance $\omega_{\text {nas }}$, while at lower frequencies $\left(\omega_{n}<0.9\right)$ it is a leaky wave radiating into a dielectric layer with the higher permittivity $\varepsilon_{1}$.

In contrast to the mode $\mathrm{B}$, the SPP mode $\mathrm{A}$ is a bound wave confined to the film interface at any frequency, and its attenuation is strongly affected by losses as illustrated by the plots of $\operatorname{Im} \gamma_{n}$ at several values of $v_{n}$ in Fig. 5. At its high frequency cut-off $\omega_{n} \approx \omega_{\text {nas } 1}<\omega_{\text {nas }}$, the SPP mode A turns into a complex mode $\mathrm{G}$ existing in the bandgap $\omega_{\text {nas } 1}<\omega_{n}<\omega_{\text {nas. }}$. The field and Poynting vector distributions [28] show that the mode $G$ remains the bound complex wave despite its dispersion curve at $\omega_{n} \geq \omega_{\text {nas } 1}$ resembles the one for the complex leaky mode $\mathrm{D}$ in the symmetric structure. As frequency increases, the mode $G$ evolves into the higher order complex bound mode associated with the plasmonic resonance $\omega_{\text {nas }}$. As the result, its fields and power flux are displaced from the interface at $y=a_{0} / 2$ (dielectric layer with higher permittivity $\varepsilon_{1}$ ) to the opposite interface $y=-a_{0} / 2$ (dielectric layer of lower permittivity $\varepsilon_{2}$ ). This field redistribution is accompanied by the increased transverse power flow and higher attenuation of the mode G. At $\omega_{n}>\omega_{\text {nas }}$, the mode $\mathrm{G}$ converts into the next higher order complex mode $\mathrm{D}$ as illustrated by $\operatorname{Im} \gamma_{n}$ in Fig. 5 .

It is important to note in Fig. 5 that $\left|\operatorname{Im} \gamma_{n}\right|$ of the forward modes $\mathrm{A}$ and $\mathrm{B}$ grows with losses (larger $v_{n}$ ), but the effect of $v_{n}$ on the complex backward modes $\mathrm{G}$ and $\mathrm{F}$ is opposite. This phenomenon is apparently related to the fact that losses are accrued to damping in forward waves but reduce the extinction rate of backward waves as can be inferred from the alternative form (8) of the DE.

Thickness $a_{0}$ of the lossy semiconductor film has significant impact on the mode properties in the vicinity of plasmonic resonances and bandgap. This effect is particularly notable in the case of asymmetric structure where the plasmonic resonances $\omega_{\text {nas } 1}$ and $\omega_{\text {nas }}$ differ. Analysis of the dispersion and attenuation characteristics and the respective field and Poynting vector distributions in the structures with dissimilar dielectrics has shown that in thicker films the SPP mode B becomes a bound wave at $\omega_{n}<\omega_{\text {nas. }}$. In the films with $a_{0} \sim 100 \mu \mathrm{m}$, the mode B has a cut-off at $\omega_{n}=\omega_{\text {nas }}$ where it exhibits a strong resonance. These results indicate that interaction of the interfaces in thick semiconductor films is rather weak, and SPP modes A and $\mathrm{B}$ are well localised at the opposite interfaces.

\subsubsection{Dielectric layers with dissimilar thicknesses $\left(a_{1} \neq a_{2}\right)$}

Asymmetry of the canonical structure in Fig. 1 with the dielectric layers of thicknesses $a_{1} \neq a_{2}$ significantly affects the properties of eigenwaves. As shown in Section 4.1, the SPPs modes $\mathrm{O}^{-}$and $\mathrm{C}^{\prime}$ in such lossless structures may expand into the bandgap $\omega_{\text {nas } 1}<\omega_{\text {nas }}<\omega_{n}<1$, and the SPP modes $\mathrm{O}^{+}$and $\mathrm{O}^{-}$may "collide" (Fig. 3) when thickness $a_{2}$ of the dielectric layer is commensurate with the scale of the SPP field localisation at the film interface. Since even small losses in thin films represent singular perturbations in vicinities of the plasmonic resonances, the eigenwave spectrum may be qualitatively altered as shown in Section 4.2. Therefore it is necessary to examine how the properties of the eigenmodes in the asymmetric structures are affected by losses at various thicknesses of dissimilar dielectric layers $\left(\varepsilon_{1}=9, \varepsilon_{2}=3.9\right)$.

The dispersion and attenuation characteristics for $a_{2}=40 \mu \mathrm{m}$ and variable $a_{1}=2,5$ and $40 \mu \mathrm{m}$ are shown in Fig. 6 . The SPP mode $\mathrm{A}$ is predominantly localised near the interface at $y=a_{0} / 2$ and experiences cut-off at the plasmonic resonance $\omega_{\text {nas } 1}$. This implies that a thinner dielectric layer $a_{1}$ causes a closer confinement of the field to the film that entails increase of both $\operatorname{Re} \gamma_{n}$ and $\operatorname{Im} \gamma_{n}$ of the modes A, D and G. At $\omega_{n a s 1}$ the mode A converts in either the complex bound mode $\mathrm{G}$ or leaky mode $\mathrm{D}$, depending on the relative rate of the field decay near the film interface. Similar effect has been observed in the semiconductor films of different thicknesses $a_{0}$, discussed in Section 4.3.1. Thus when $a_{1}$ becomes very small, $a_{1}<<a_{0}$, e.g. $a_{1}=2 \mu \mathrm{m}$ in Fig. 6 , the bound mode A turns directly into the leaky mode D which radiates into dielectric layer with higher permittivity $\varepsilon_{1}$ at frequencies $\omega_{n}>\omega_{\text {nas } 1}$. Therefore at $a_{1}<<a_{0}$, similarly to the case of thick semiconductor film, the plasmonic resonance $\omega_{\text {nas }}$, associated with the other dielectric layer, has little influence on 

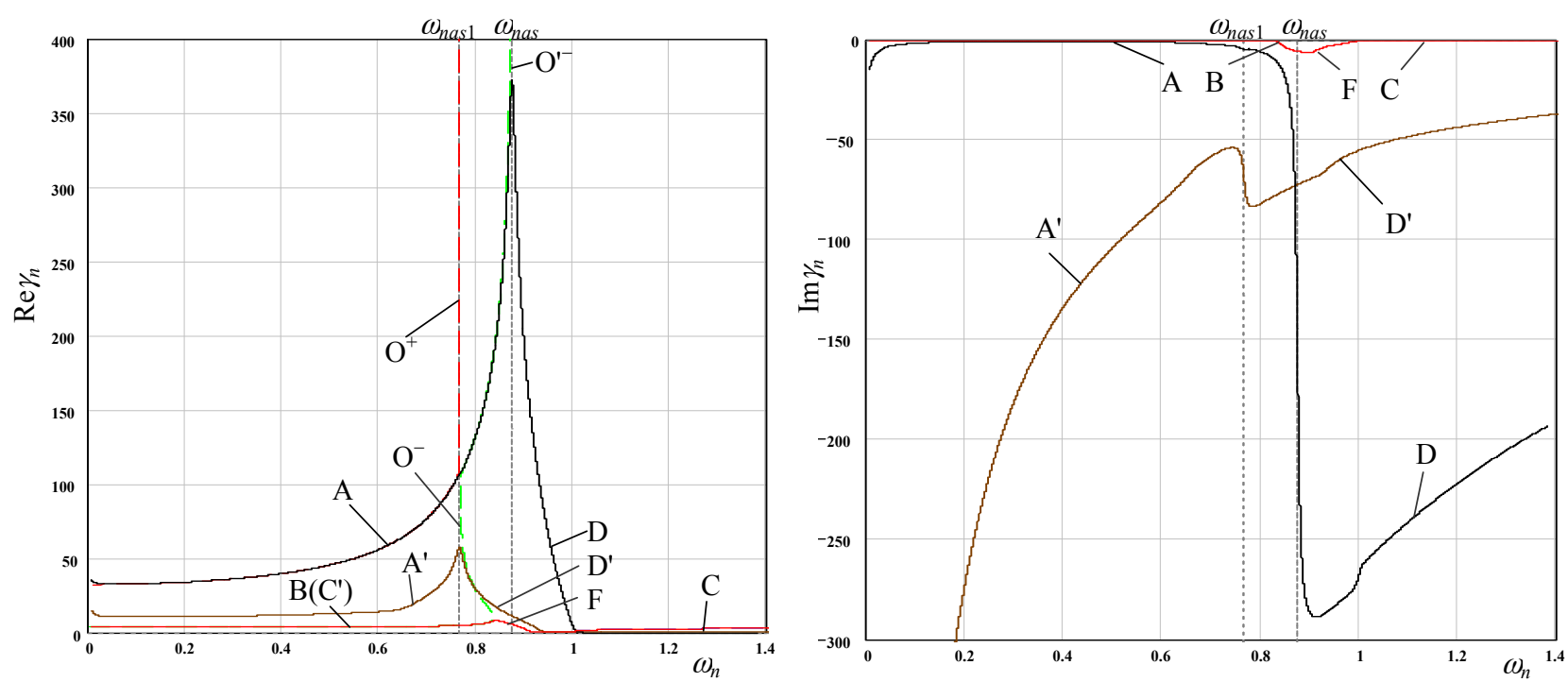

Fig. 7. Propagation $\left(\operatorname{Re} \gamma_{n}\right)$ and attenuation $\left(\operatorname{Im} \gamma_{n}\right)$ constants of eigenmodes in the asymmetric structure with the parameters: $a_{0}=20 \mu \mathrm{m}, a_{1}=40 \mu \mathrm{m}$, $a_{2}=2 \mu \mathrm{m}, \varepsilon_{1}=9, \varepsilon_{2}=3.9, \varepsilon_{L}=13.1, v_{n}=0.01, \omega_{p}=848.17 \times 10^{9} \mathrm{rad} / \mathrm{sec} . \mathrm{O}^{-}, \mathrm{O}^{+}, \mathrm{C}^{\prime}$ and $\mathrm{C}$ are the modes in the lossless structure $\left(v_{n}=0\right) ; \mathrm{A}, \mathrm{B}, \mathrm{C}, \mathrm{D}$ and $\mathrm{F}$ are the fundamental SPP eigenmodes in presence of loss $\left(v_{n}=0.01\right)$. $\mathrm{A}^{\prime}$ and $\mathrm{D}^{\prime}$ are the lowest order complex SPP modes.

the mode $\mathrm{D}$, and no resonance kink is observed at $\omega_{n}=\omega_{\text {nas }}$ on the dispersion curve for $a_{1}=2 \mu \mathrm{m}$ in Fig. 6 . In contrast to the mode A, the SPP mode B is less sensitive to variations of $a_{1}$, being mostly confined to the opposite interface of the semiconductor film at $y=-a_{0} / 2$.

Thickness $a_{2}$ of the dielectric layer with lower permittivity $\varepsilon_{2}$ has a profound effect on the properties of the SPP modes $\mathrm{O}^{-}$and $\mathrm{O}^{+}$in the lossless structure. As shown in Section 4.1, when the layer of thickness $a_{2}$ becomes thinner $\left(a_{2} \sim 1 \div 2 \mu \mathrm{m}\right.$ ), the modes $\mathrm{O}^{-}$and $\mathrm{O}^{+}$can "collide" (see Fig. 3) and interchange their location at the film interfaces. To examine this effect in presence of loss, the characteristics of the fundamental and higher order SPP modes have been simulated for the dielectric layers with $a_{1}=40 \mu \mathrm{m}, a_{2}=2 \mu \mathrm{m}$ and $\varepsilon_{1}=9, \varepsilon_{2}=3.9$. The dispersion characteristics of the SPP modes $\mathrm{A}, \mathrm{B}$ and $\mathrm{D}^{\prime}$ in the lossy structure and respective modes $\mathrm{O}^{+}, \mathrm{O}^{-}, \mathrm{O}^{\prime-}$ and $\mathrm{C}^{\prime}$ in the lossless case are juxtaposed in Fig. 7. Comparison of Fig. 6 and Fig. 7 shows that when thickness $a_{2}$ decreases, $\operatorname{Re} \gamma_{n}$ of the mode A grows similarly to the case of thinner layer $a_{1}$ as discussed above. Besides the fields of the mode A are displaced to the opposite interface of the semiconductor film toward the dielectric layer of lower permittivity $\varepsilon_{2}=3.9\left(<\varepsilon_{1}=9\right)$. This implies that $\gamma_{n}\left(\omega_{n}\right)$ of the mode A should tend asymptotically to the higher frequency plasmonic resonance $\omega_{\text {nas }}$. In the lossless case, this has led to "collision" of the modes $\mathrm{O}^{-}$and $\mathrm{O}^{+}$at $\omega_{n}=\omega_{\text {nas } 1}$, see Fig. 3. The plots in Fig. 7 for the lossy film show that $\operatorname{Re} \gamma_{n}$ of the mode A remains continuous at $\omega_{n}=\omega_{n a s 1}$ and follows the respective curves for the mode $\mathrm{O}^{+}$at $\omega_{n}<\omega_{\text {nas } 1}\left(\operatorname{Re} \gamma_{n}<\operatorname{Re} \gamma_{n}\left(\omega_{\text {nas } 1}\right)\right)$ and the mode $\mathrm{O}^{\prime-}$ at $\omega_{n a s 1}<\omega_{n}<\omega_{\text {nas }}\left(\operatorname{Re} \gamma_{n}>\operatorname{Re} \gamma_{n}\left(\omega_{\text {nas } 1}\right)\right)$. While the curve $\operatorname{Re} \gamma_{n}\left(\omega_{n}\right)$ is smooth at $\omega_{n}=\omega_{\text {nas } 1}, \operatorname{Im} \gamma_{n}\left(\omega_{n}\right)$ has a small kink. This indicates that the effect of the mode "collision" at $\omega_{n}=\omega_{\text {nas } 1}$ still occurs but the mode A does not exhibit a resonant absorption at $\omega_{\text {nas } 1}$. The simulated field distributions have confirmed that the dominant polarisation of the mode A, indeed, flips at $\omega_{n}=\omega_{n a s}$, whilst the mode A still remains localised at $y=-a_{0} / 2$, near the interface with the dielectric layer of lower permittivity $\varepsilon_{2}$.

The other modes involved with the mode "collision" in the lossless case are suppressed by the film losses. Fig. 7 shows that the leaky complex mode D' appears instead of the backward mode $\mathrm{O}^{-}$with $\operatorname{Re} \gamma_{n}<\operatorname{Re} \gamma_{n}\left(\omega_{\text {nas } 1}\right)$ in the bandgap $\omega_{\text {nas } 1}<\omega_{n}<\omega_{\text {nas }}$. The mode $\mathrm{D}^{\prime}$ evolves from the higher order bound complex mode $\mathrm{A}^{\prime 3}$. The complex mode corresponding to the SPP mode $\mathrm{O}^{+}$with $\operatorname{Re} \gamma_{n}>\operatorname{Re} \gamma_{n}\left(\omega_{n a s 1}\right)$ has so strong attenuation that its $\operatorname{Im} \gamma_{n}$ is beyond the scale of Fig. 7.

Unlike the mode A and A', the long-range SPP mode B is weakly perturbed by variations of $a_{2}$ (see Fig. 7), similarly to the respective mode $C^{\prime}$ in the lossless structure. Being primarily localised at the opposite film interface $\left(y=a_{0} / 2\right)$, the mode $\mathrm{B}$ exhibits the resonance at $\omega_{n c}\left(\omega_{n c}>\omega_{n a s 1}\right)$, which corresponds to the cut-off frequency of the modes $\mathrm{C}^{\prime}$ and $\mathrm{O}^{-}$ in the lossless structure. At $\omega_{n}=\omega_{n c}$ the mode B converts into the bound complex mode F.

Thus, the presented analysis shows that both permittivity and thickness of the dielectric layers strongly affect the spectrum and properties of the SPP waves in the lossy semiconductor films. The simulation results have demonstrated that only forward type SPP modes are the bound waves guided by the real semiconductor films, while the complex and leaky waves with negative slope of the dispersion curves are strongly attenuated.

\footnotetext{
${ }^{3}$ The complex mode $\mathrm{A}^{\prime}$ exists along with the SPP modes A and B in the frequency band $\omega_{n}<\omega_{\text {nas } 1}$ and experiences a resonance at $\omega_{n}=\omega_{\text {nas } 1}$ where it converts into the complex leaky mode D'.
} 


\section{Surface plasmons in Au film on glass substrate}

The analysis of eigenwaves in the semiconductor films has been performed for Drude permittivity $\varepsilon_{s}(\omega)$ defined by (1). While the Drude model provides an adequate description of semiconductor plasma at mm-wave and $\mathrm{THz}$ frequencies, its accuracy is limited in the optical range. Therefore the measured optical constants of metals available in the tabulated form, e.g. [21], can be used instead. Recently, the SPP propagation in thin silver film has been investigated numerically in [20] using the measured optical constant of Ag. An alternative approach proposed in [17] is adopted in this paper to examine the properties of eigenwaves in thin $\mathrm{Au}$ film on glass substrate enclosed in a parallel-plate waveguide (Fig. 1). The optical permittivity of Au is given by the table data [21].

In order to take benefit of the asymptotic solutions and numerical analysis presented in Sections 3, 4 for the SPP in semiconductor films, it is convenient to interrelate the Drude model parameters $\omega_{p}$ and $v$ with the table data for the Au permittivity. Making use of the $\varepsilon_{s}(\omega)$ definition in (1) and taking into account that $\varepsilon_{L}=1$ for metals, $\omega_{p}$ and $v$ can be expressed in terms of the complex permittivity $\varepsilon_{A u}(\omega)=\varepsilon_{A u}^{\prime}(\omega)+i \varepsilon_{A u}^{\prime \prime}(\omega)$ at any given frequency $\omega$

$$
v(\omega)=\frac{\varepsilon_{A u}^{\prime \prime}(\omega)}{1-\varepsilon_{A u}^{\prime}(\omega)} ; \quad \omega_{p}(\omega)=\sqrt{\left[1-\varepsilon_{A u}^{\prime}(\omega)\right]\left[\omega^{2}+v^{2}(\omega)\right]} .
$$

It is important to note that $\omega_{p}(\omega)$ and $\gamma(\omega)$ are the frequency dependent quantities here because in a broad frequency range the actual dispersion of Au permittivity deviates from that given by the Drude model. Since both $\omega_{p}(\omega)$ and $v(\omega)$ are slowly varying functions as illustrated by Fig. 8, they can be utilised for the numerical solution of the DE (2).

Eigenwaves in the Au films have been analysed in the frequency range $242-880 \mathrm{THz}$ where the permittivity given by the table data [21] is negative, i.e. $\varepsilon_{A u}^{\prime}<0$. The characteristics of the fundamental mode in $\mathrm{Au}$ films of thicknesses $a_{0}=25$ and $50 \mathrm{~nm}$ on glass substrate with permittivity $\varepsilon_{1}=1.5$ are shown in Fig. 9 for several thicknesses of the substrate $\left(a_{1}\right)$ and a layer of air $\left(a_{2}\right)$. At all considered thicknesses of the dielectric layers, $\operatorname{Re} \gamma_{n}$ has a peak at frequency $f_{d} \approx 593 \mathrm{THz}$. This peak is usually attributed to the plasmonic resonance in lossy $\mathrm{Au}$ film. The peak is sharper in thinner Au films, whilst $\gamma_{n}$ exhibits rather weak dependence on the dielectric layer thicknesses. However a considerable offset of the $\operatorname{Re} \gamma_{n}$ peak from the $\operatorname{Im} \gamma_{n}$ dip in Fig. 9 at a relatively low magnitude of $\operatorname{Re} \gamma_{n}$ is hardly consistent with the conventional SPP characteristics. In order to understand such behaviour of SPP modes in Au film and duly assess the role of loss, the dispersion characteristics have been evaluated at a few values of $v_{n}(\omega)$, viz. the actual $v_{n}(\omega)=$ $v(\omega) / \omega_{p}(\omega)$, retrieved from the table data, and $v_{n}(\omega)$ scaled by the factors $10^{-3}, 10^{-2}, 0.1$, and 0.5 . Comparison of the obtained results is presented in Fig. 10 for the structure

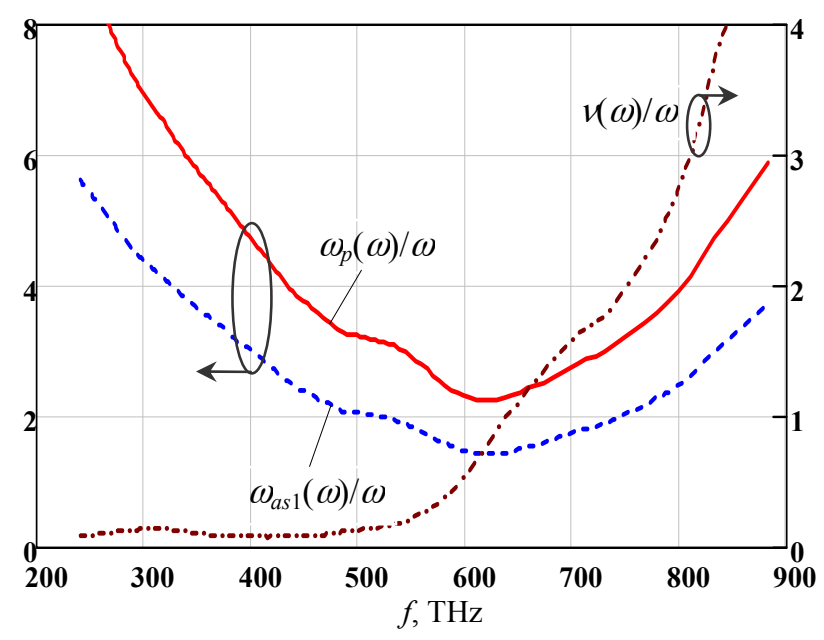

Fig. 8. Plasma frequency $\omega_{p}(\omega)$ and collision frequency $v(\omega)$, retrieved from the table data for $\mathrm{Au}$, and plasmonic resonance frequency $\omega_{a s 1}(\omega)$ all normalised to angular frequency $\omega=2 \pi f$. with the parameters: $\varepsilon_{1}=1.5, \varepsilon_{2}=1, a_{0}=a_{1}=a_{2}=50 \mathrm{~nm}$.

Examination of Fig. 10 shows that while the attenuation constant $\operatorname{Im} \gamma_{n}$ varies proportionally to the scaled $v_{n}$, the respective changes of $\operatorname{Re} \gamma_{n}$ are very marginal. Moreover, instead of the anticipated sharp rise of the $\operatorname{Re} \gamma_{n}$ peak, which would be expected near the plasmonic resonance as the scaling factor of $v_{n}$ decreases from 0.1 to $10^{-3}$, the effect on Re $\gamma_{n}$ is hardly discernible. This suggests that the peak of $\operatorname{Re} \gamma_{n}$ is not directly related to the plasmonic resonance but has a different nature. Indeed, according to (6), the plasmonic resonance frequency $\omega_{a s 1}$ is proportional to $\omega_{p}(\omega)$, which varies with frequency for $\varepsilon_{A u}(\omega)$ given by the table data [21], cf. (9). Thus the necessary condition of the plasmonic resonance to occur is $\omega=\omega_{a s 1}(\omega)$. However inspection of Fig. 8 shows that this condition cannot be satisfied at any frequency because $\omega_{a s 1}(\omega)>1.5 \omega$ in the entire frequency range. Therefore the peak of $\operatorname{Re} \gamma_{n}$ in Fig. 9 and Fig. 10 has its origins in the non-Drude dispersion of $\varepsilon_{A u}(\omega)$ at optical frequencies that restrains the attainable values of $\operatorname{Re} \gamma_{n}$. Indeed, the peak of $\operatorname{Re} \gamma_{n}$ at $f_{d} \approx 593 \mathrm{THz}$ is correlated with the minimum of $\omega_{a s 1}(\omega) / \omega$, see Fig. 8. Then as $\omega_{a s 1}(\omega) / \omega$ grows at $f>f_{d}$, the frequency separation $\left(\omega_{a s 1}(\omega)-\omega\right)$ increases that leads to the fall of $\operatorname{Re} \gamma_{n}$, which resembles the dispersion curve of backward wave. The field and Poynting vector distributions in Fig. 11 corroborate this interpretation of the SPP behaviour and demonstrate that SPP mode remains a forward wave with the wave vector and total power flow being codirectional. It is necessary to note that $v_{n}(\omega) / \omega$ grows at $f>f_{d}$ as well, and this causes the observed offset between the peaks of $\operatorname{Re} \gamma_{n}$ and dip of $\operatorname{Im} \gamma_{n}$, cf. Fig. 9 and Fig. 10. 

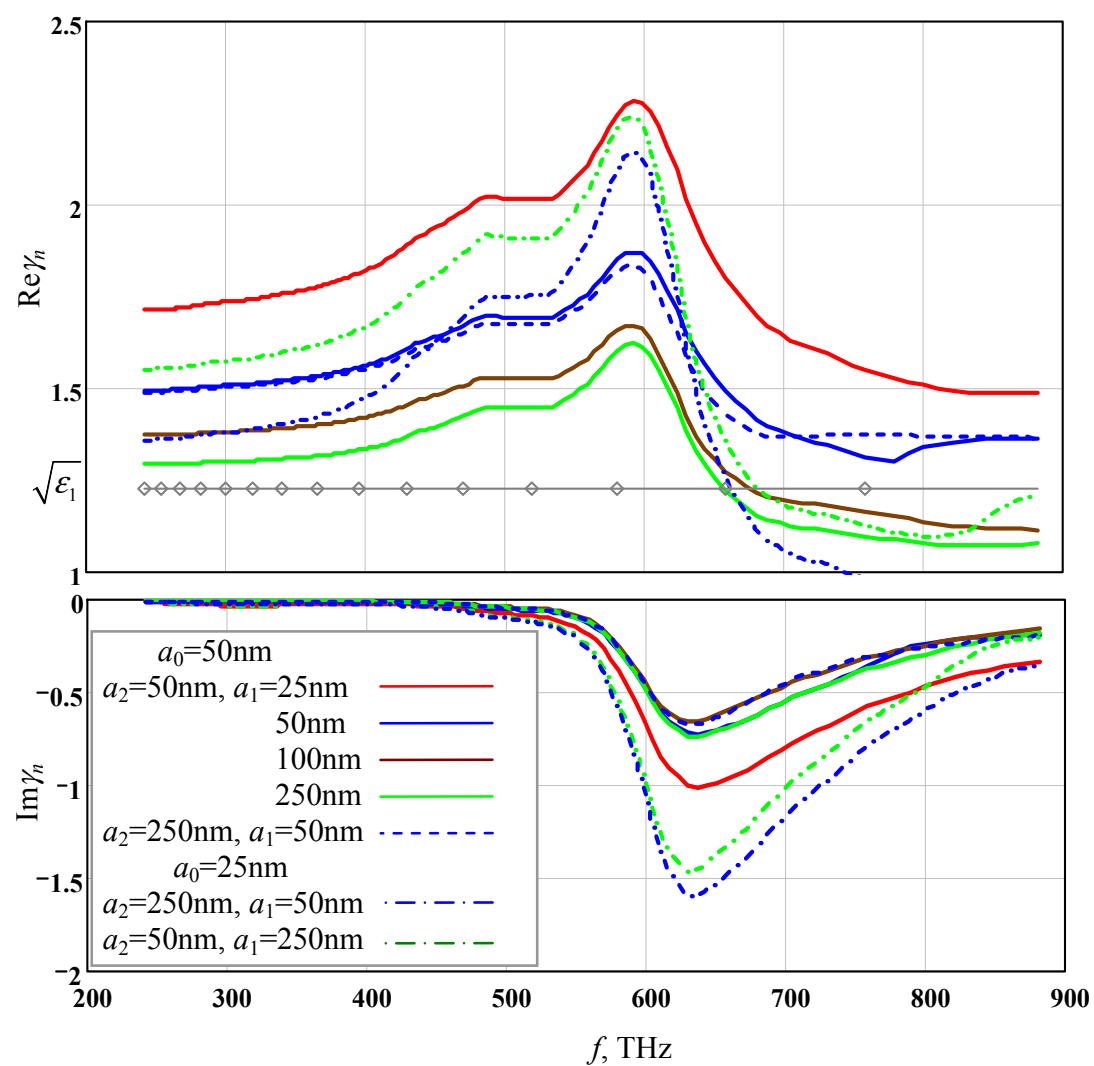

Fig. 9. Propagation $\left(\operatorname{Re} \gamma_{n}\right)$ and attenuation $\left(\operatorname{Im} \gamma_{n}\right)$ constants of the fundamental mode in the $\mathrm{Au}$ film of thickness $a_{0}$ on glass substrate of thickness $a_{1}$ and permittivity $\varepsilon_{1}=1.5$; the layer of air (permittivity $\varepsilon_{2}=1$ ) has thickness $a_{2}$.

To elucidate the properties of the fundamental SPP mode propagating in $\mathrm{Au}$ film, the normalised distributions of fields and Poynting vector in the waveguide cross-section are presented in Fig. 11 at frequencies $f=486,591,776 \mathrm{THz}$ (the structure parameters are the same as in Fig. 10). Inspection of the plots in Fig. 11 shows that the fundamental mode has the main notions of a bound SPP with the power flux $\left(\operatorname{Re} P_{z}\right)$ localised at the film interfaces and the dominant longitudinal polarisation $\left|E_{z}\right|>\left|E_{y}\right|$ inside the $\mathrm{Au}$ film. However outside the film this ratio is inverse, viz. $\left|E_{z}\right|<\left|E_{y}\right|$ at $|y|>a_{0} / 2$. This implies that the fundamental mode in the $\mathrm{Au}$ film significantly differs from the ordinary long-range SPP. Further examination of the Poynting vector distributions shows that at lower frequencies, $f<f_{d}$, the power flow confinement to the glass substrate interface increases with frequency, cf. rows (a) and (b) in Fig. 11, and $\operatorname{Re} P_{z}$ in the $\mathrm{Au}$ film and surrounding dielectric layers are counter-directed, similarly to SPP in semiconductor films, as discussed in Section 4. However at $f>f_{d}$ where $\omega_{a s 1}(\omega) / \omega$ grows, the field confinement decreases, and the power flow is primarily localised outside of the $\mathrm{Au}$ film. Despite the dispersion curves exhibit a negative slope at $f>f_{d}$ that could suggest negative group velocity $v_{g}=d \omega / d\left(\operatorname{Re} \gamma_{n}\right)$ of SPP, the energy is predominantly transported in the dielectric layers and the total power flow remains codirectional with the wave vector. This demonstrates again that the group velocity inferred from the dispersion
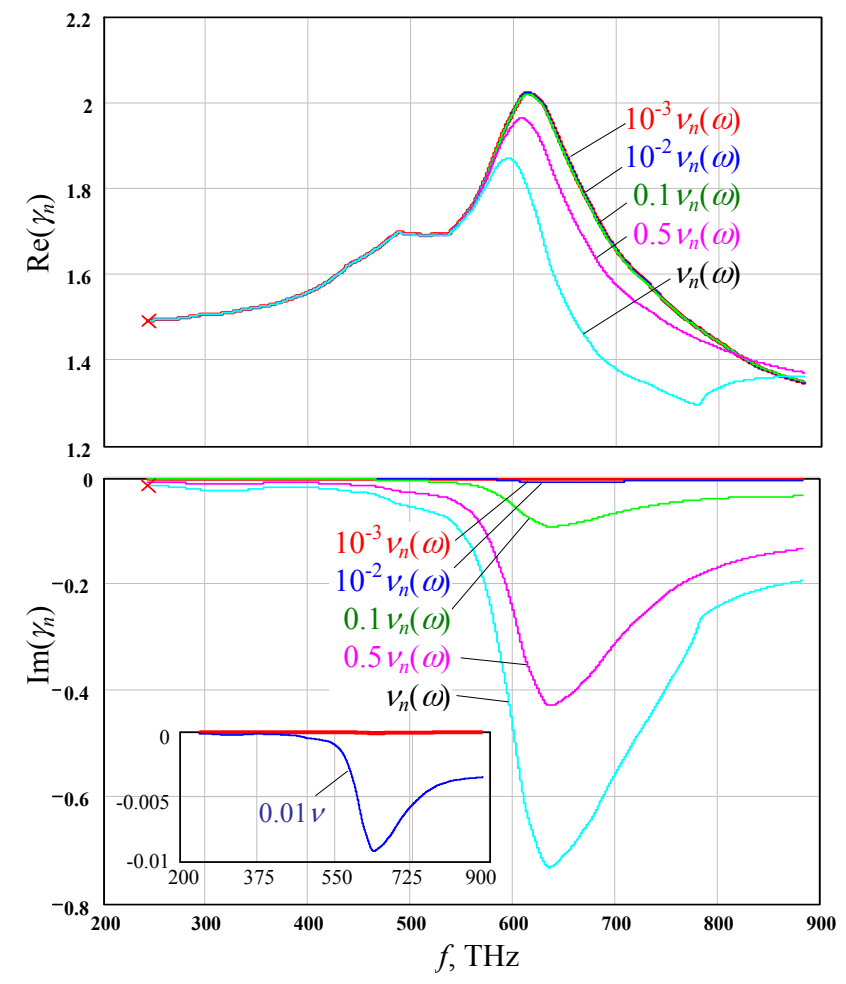

Fig. 10. Propagation $\left(\operatorname{Re} \gamma_{n}\right)$ and attenuation $\left(\operatorname{Im} \gamma_{n}\right)$ constants vs. scaled $v_{\mathrm{n}}(\omega)$ for the fundamental SPP mode in Au film on glass substrate in the layered waveguide with the following parameters: $\varepsilon_{1}=1.5, \varepsilon_{2}=1, a_{0}=a_{1}=$ $a_{2}=50 \mathrm{~nm} ; v_{\mathrm{n}}(\omega)$ retrieved from the table data is additionally scaled by the factors $10^{-3}$ (Red), $10^{-2}$ (Blue), 0.1 (Green), and 0.5 (Pink); $\omega=2 \pi f$. 

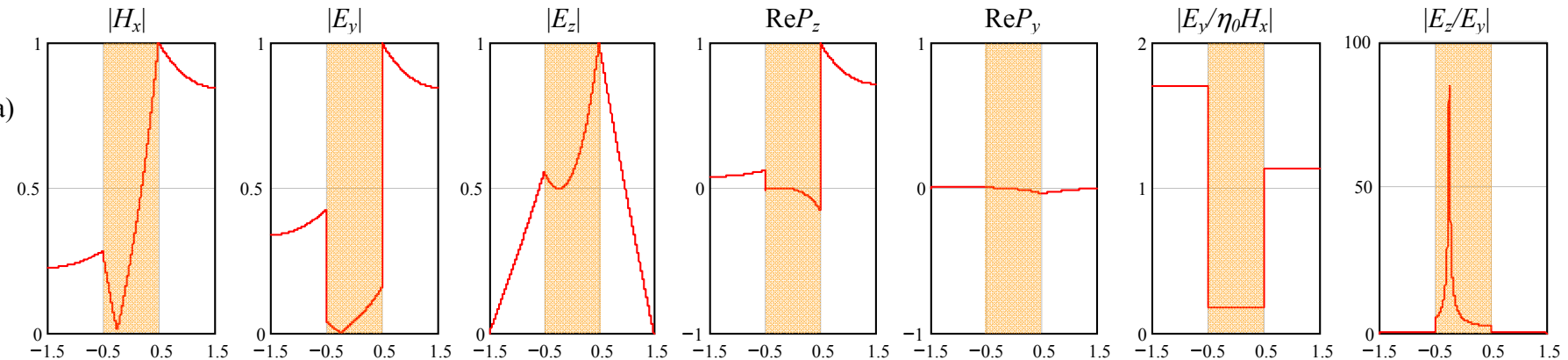

(b)
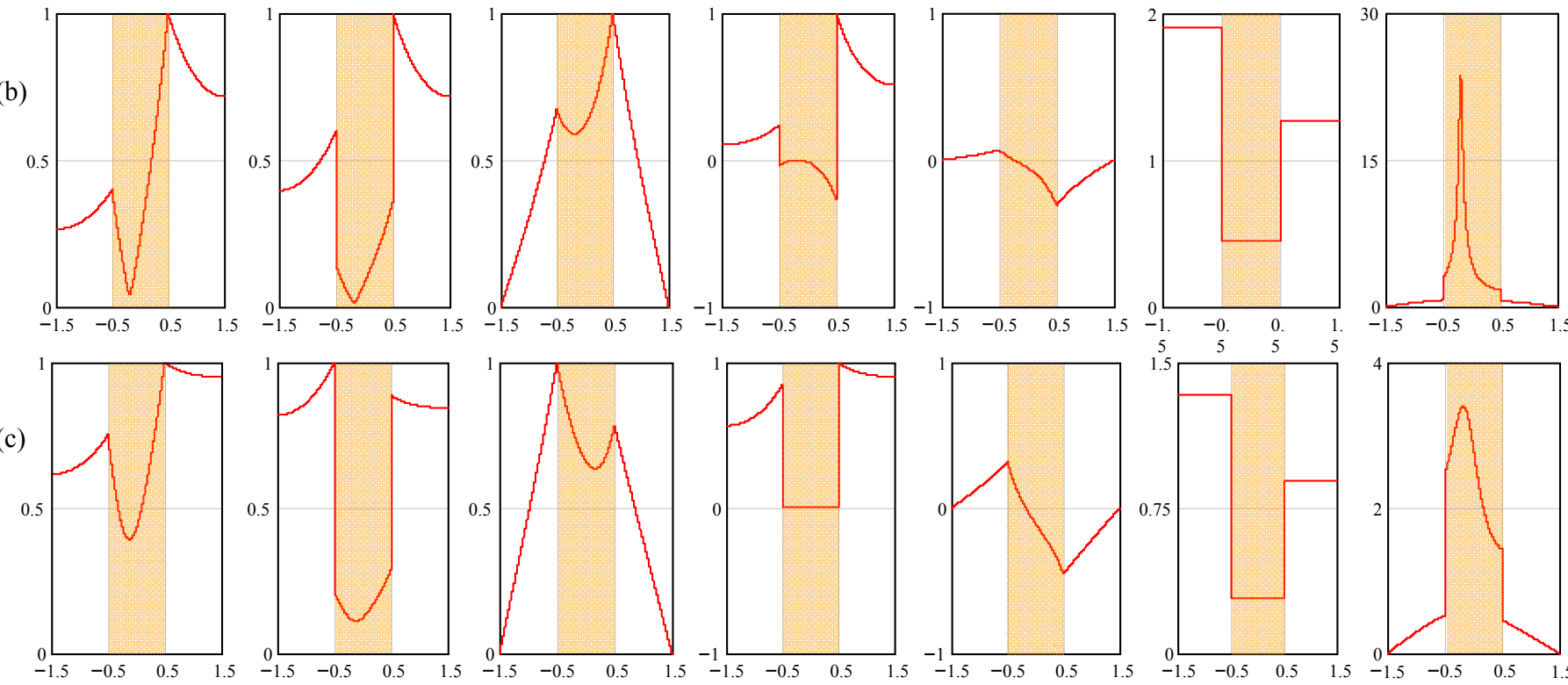

$y / a_{0}$

Fig. 11. Normalized cross-sectional distributions of fields $H_{x}, E_{y}, E_{z}$, Poynting vector $P_{z}, P_{y}$, wave impedance $\left|E_{y} / \eta_{0} H_{x}\right|$ and polarisation $\left|E_{z} / E_{y}\right|$ at (a): $f=486 \mathrm{THz}, \gamma_{n}=1.69-i 0.048 ;$ (b): $f=591 \mathrm{THz}, \gamma_{n}=1.87-i 0.38$; (c): $f=776 \mathrm{THz}, \gamma_{n}=1.29-i 0.3$. The Au film is located at $|y|<a_{0} / 2$ (shaded area).

characteristics of waves with strong dispersion and attenuation is no representative of the power flow direction as mentioned earlier, cf. [30]. Thus the fundamental mode guided by the Au film represents the SPP distorted by the actual frequency dispersion of $\varepsilon_{A u}(\omega)$, which determines the SPP properties in the Au film and limits the maximum attainable value of the slow wave factor $\operatorname{Re} \gamma_{n}$.

\section{Conclusions}

Transverse magnetic (TM) waves have been explored in the canonical structure of layered waveguide with lossy semiconductor and metallic films sandwiched between two dielectric layers. The Drude model and table data of the optical constants have been used to describe the permittivity of semiconductor and Au films, respectively. The effects of loss, geometry and the parameters of dielectric layers on the eigenmode spectrum and, particularly, on the SPP have been analysed using both the asymptotic and rigorous numerical solutions of the full-wave dispersion equation. To identify the modes and elucidate the main properties of the eigenwaves in semiconductor and Au films, their fields and Poynting vector distributions have been simulated. It has been shown that the complex waves constitute an essential part of the eigenmode spectrum in the thin film waveguides, and variations of the structure symmetry and parameters of the dielectric layers may cause the qualitative changes of the SPP properties and the whole spectrum. The main features of SPP modes in the lossy semiconductor films can be summarised as follows.

- At $\left|\gamma_{n}\right| \gg>1$, the SPP attenuation constant $\operatorname{Im} \gamma_{n}$ grows at the much higher rate of $\sim v\left(\operatorname{Re} \gamma_{n}\right)^{3}$ than that of $\sim v \operatorname{Re} \gamma_{n}$ for the dynamic waves. This implies that even small losses introduce singular perturbations of SPP near the plasmonic resonances and cause strong attenuation of SPP even at small values of $v$.

- The two fundamental eigenwaves, usually referred to as the short-range (mode A) and long-range (mode B) SPP, have even and odd distributions of their longitudinal electric field $E_{z}$ in the symmetric structure, respectively. The mode A exist only at frequencies $\omega_{n}<\omega_{\text {nas }}$, while the mode B can expand also into the bandgap $\left(\omega_{\text {nas }}<\omega_{n}<1\right)$ where it experiences a cut-off at $\omega_{n}=\omega_{n c}\left(\omega_{n a s}<\omega_{n c}<1\right)$ and its $\operatorname{Re} \gamma_{n}$ reaches the maximum.

- In asymmetric structures, the field of the bound modes A and B are localised predominantly at opposite interfaces of the film. At low frequencies $\omega_{n}<\omega_{n b}$, the SPP mode B is a leaky wave radiating into a dielectric layer with higher 
permittivity and it becomes a bound wave only in a narrower band $\omega_{n b}<\omega_{n}<\omega_{n c}$. The layer aspect ratio and permittivity contrast determine the transition frequency $\omega_{n b}$ which may fall in the bandgap, i.e. $\omega_{n b}>\omega_{\text {nas }}$. In the latter case, the frequency bands, where the modes A and B are the bound waves, are separated.

- Both SPP modes A and B are forward waves with positive dispersion at any frequency except the low frequency limit where they experience strong attenuation. In contrast to the spectrum of the lossless semiconductor film, no backward wave representing the "loss perturbed" SPP mode $\mathrm{O}^{-}$with $\operatorname{Re} \gamma_{n}>\operatorname{Re} \gamma_{n}\left(\omega_{n c}\right)$ has been found in the frequency band $\omega_{\text {nas } 1}<\omega_{n}<\omega_{n c}$. Instead, the complex leaky mode D appeared to be the lowest order mode, whose $\operatorname{Re} \gamma_{n}$ closely follows the dispersion curve of the backward SPP mode $\mathrm{O}^{-}$.

- The complex modes G, D and F resemble backward waves with negative dispersion $\left(d \omega_{n} / d \operatorname{Re} \gamma_{n}<0\right)$. However their total power flows $\operatorname{Re} P_{z}$ over the entire waveguide cross-section remain co-directional with the wave vector. These results demonstrate that the group velocity direction determined by the slope of $\operatorname{Re} \gamma_{n}$ is no representative of the direction of the total power flow of complex or strongly attenuated SPP modes.

- The SPP mode "collision" observed in the lossless structure with a thin dielectric layer $\left(a_{2}<<a_{0}\right)$ of lower permittivity $\left(\varepsilon_{2}<\varepsilon_{1}\right)$ manifests itself in the abrupt change of the mode A polarisation without strong resonant absorption at $\omega_{n}=\omega_{\text {nas }}$ in the lossy semiconductor film. The field and Poynting vector distributions demonstrate that the dominant polarisation of the mode A flips at $\omega_{n}=\omega_{\text {nas }}$, whilst the mode A preserves the main traits of the longrange SPP in the expanded frequency band $\omega_{\text {nas } 1}<\omega_{n}<\omega_{\text {nas }}$.

- Poynting vector $\left(\operatorname{Re} P_{z}, \operatorname{Re} P_{y}\right)$ distributions of SPP have shown that the longitudinal power fluxes $\operatorname{Re} P_{z}$ inside and outside of the semiconductor film are counter-directed, while the transverse power flow $\operatorname{Re} P_{y}$ points into the film. These patterns of the SPP Poynting vectors can be interpreted as vortices of the power flow, which trap a portion of the total power carried by SPP. Such a mechanism of energy transport by SPP provides a comprehensive interpretation of the SPP characteristics and properties. In particular, the vorticity of the SPP Poynting vector is unambiguously correlated with the dispersion and attenuation properties of the SPP modes and the anomalously high attenuation rate of $\operatorname{Im} \gamma_{n} \sim v\left(\operatorname{Re} \gamma_{n}\right)^{3}$ at $\operatorname{Re} \gamma_{n}>>1$.

Eigenwaves in $\mathrm{Au}$ films on a glass substrate $\left(\varepsilon_{1}=1.5\right)$ have been analysed in the optical range where the $\mathrm{Au}$ permittivity, given by the table data [21], has negative $\operatorname{Re} \varepsilon_{A u}(\omega)$. The presented results have conclusively demonstrated that the maximal attainable slow wave factor $\operatorname{Re} \gamma_{n}$ of SPP guided by the Au films is limited not by losses but the actual dispersion of $\varepsilon_{A u}(\omega)$. The non-Drude dispersion of $\varepsilon_{A u}(\omega)$ precludes the fundamental SPP mode from reaching the plasmonic resonance, and the peak of $\operatorname{Re} \gamma_{n}$ observed at $f_{d} \approx 593 \mathrm{THz}$ corresponds to the minimal frequency offset from the plasmonic resonance $\omega_{a s 1}(\omega)$. The described properties of the fundamental SPP mode in Au film have been corroborated by the fields and Poynting vector distributions in the waveguide cross-section.

\section{Acknowledgement}

This work was supported by the International Centre for System-on-Chip and Advanced Microwireless Integration (SoCaM), Queen's University Belfast, UK.

\section{References}

[1] "Surface polaritons: electromagnetic waves at surfaces and interfaces", Ed. V.M. Agranovich, D.L. Mills, NorthHolland, 1982.

[2] "Electromagnetic Surface Modes", Ed. A. D. Boardman, Wiley, 1982.

[3] D.L. Mills, A.A. Maradudin, "Properties of surface polaritons in layered structures", Phys. Rev. Lett. v. 31, pp.372-375, 1973.

[4] J. S. Nkoma, R. Loudon and D. R. Tilley, "Elementary properties of surface polaritons", J. Phys. C, v.7, pp. 35473559,1974

[5] D. Sarid, "Long-range surface-plasma waves on very thin metal flims", Phys. Rev. Lett. v.47, pp.1927-1930, 1981.

[6] G. I. Stegman, J. J. Burke and D. G. Hall, "Surface-polariton-like waves guided by thin, lossy metal films,", Opt. Lett. 8, pp. 383-385, 1983.

[7] J. J. Burke, G. I. Stegeman \& T. Tamir, "Surface polariton like waves guided by thin, lossy metal films", Phys. Rev. B., Vol. 33, pp. 5186 - 5201, 1986.

[8] G.J. Kovacs, "Optical Excitation of Surface Plasmon-Polaritons in Layered Media ", in "Electromagnetic Surface Modes", Ed. A. D. Boardman, Wiley, 1982.

[9] J.J. Quinn, G. Eliasson and P. Hawrylak, "Bulk and surface plasma waves in semiconducting superlattices", in "Spatial dispersion in solids and plasmas", Ed. P. Halevi, North-Holland, 1992.

[10] M. S. Kushwaha, "Plasmons and magnetoplasmons in semiconductor heterostructures" Surface Science Reports, $41(2001) 1-416$.

[11] V.H. Mok, L.E. Davis, "Nonreciprocal wave propagation in multilayer semiconductor films at frequencies up to 200 GHz" IEEE Transactions on MTT, v. 51, no. 12, Dec. 2003, pp. 2453 - 2460 
[12] A.V. Zayats, I.I. Smolyaninov, A.A. Maradudin. "Nano-optics of surface plasmon polaritons", Physics Reports 408, pp. 131-314, 2005.

[13] "Nanophotonics with surface plasmons", Ed. V.M. Shalaev, S. Kawata, Elsevier, 2007.

[14] A. Yelon, K.N. Piyakis, E. Sacher, "Surface plasmons in Drude metals", Surface Science, v. 569, pp. 47-55, 2004.

[15] A. Degiron and D. Smith, "Numerical simulations of long-range plasmons," Optics Express 14, pp. $1611,2006$.

[16] R. Adato, J. Guo, "Characteristics of ultra-long range surface plasmon waves at optical frequencies", Optics Express, v. 8, pp. 5008-5017, 2007.

[17] A. G. Schuchinsky, X. Yan, "Effect of loss on spectra and properties of eigenwaves in imperfect metal films and magnetised semiconductor-dielectric structures", in Proc. "Metamaterials 2007, $1^{\text {st }}$ Int. Congress on Advanced Electromagnetic Materials in Microwaves and Optics”, Rome, Italy, Oct. 22-26, pp. 908-910, 2007

[18] P. Berini, "Plasmon-polariton waves guided by thin lossy metal films of finite width: Bound modes of symmetric structures," Phys. Rev. B 61, 10484, 2000.

[19] P. Berini, "Plasmon-polariton waves guided by thin lossy metal films of finite width: Bound modes of asymmetric structures," Phys. Rev. B 63, 125417, 2001.

[20] J. A. Dionne, L. A. Sweatlock, H. A. A. Atwater and A. Polman, "Planar metal plasmon waveguides: frequencydependent dispersion, propagation, localization, and loss beyond the free electron model", Phys. Rev. B72, 075405, 2005.

[21] E. Palik, Handbook of Optical Constants of Solids, Academic Press, NewYork, 1985.

[22] R.E. Collin, "Field Theory of Guided Waves", IEEE Press, 1991.

[23] Schuchinsky A.G., "Surface waves in tangentially magnetized ferrite slab", Radiotehnika i elektronika, v.29, No 9, pp. 1700-1704, 1984.

[24] G.A.Vugalter, "Magnetostatic waves with complex wave numbers in a ferrite film without energy losses.", Radiotehnika i elektronika, v.28, No 5, pp.955-959, 1983.

[25] V.N. Ivanov, N.P. Demchenko, I.S. Nefedov, R.A.Silin, A.G. Schuchinsky, "Waves in tangentially magnetized ferrite slab (numerical electrodynamic analysis and regular asymptotic expansions)", Izvestiya Vysshikh Uchebnykh Zavedenii. Seriya Radiofizika, v.32, No. 6, 1989, pp 764-779.

[26] P. Kravanja, M. van Barel, "A derivative-free algorithm for computing zeros of analytic functions", Computing, vol. 63 , pp. 69-78, 1999.

[27] C. J. Gillan, A. Schuchinsky and I. Spence, "Computing Zeros of Analytic Functions in the Complex Plane without using Derivatives", Computer Physics Communications (CPC), v.175, no. 4, 2006, pp. 304-313.

[28] X. Yan, "Waves in Layered Semiconductor-Dielectric Structures", PhD Thesis, Faculty of Engineering and Physical Science, Queen's University Belfast, July 2008, 244p.

[29] W. Gough, "Poynting in the wrong direction?", Eur. J. Phys. v.3, 83-87, 1982.

[30] R. Ruppin, "Electromagnetic energy density in a dispersive and absorptive material", Physics Letters A, v. 299, pp. 309-312, 2002. 